This is an electronic reprint of the original article. This reprint may differ from the original in pagination and typographic detail.

Author(s): Thompson, Marc; Diapoulis, Georgios; Himberg, Tommi; Toiviainen, Petri

Title: Interpersonal Coordination in Dyadic Performance

Year: $\quad 2017$

Version:

Please cite the original version:

Thompson, M., Diapoulis, G., Himberg, T., \& Toiviainen, P. (2017). Interpersonal Coordination in Dyadic Performance. In M. Lesaffre, P.-J. Maes, \& M. Leman (Eds.), The Routledge Companion to Embodied Music Interaction (pp. 186-194). Routledge, Taylor \& Francis Group. Routledge Companions.

https://doi.org/10.4324/9781315621364-21

All material supplied via JYX is protected by copyright and other intellectual property rights, and duplication or sale of all or part of any of the repository collections is not permitted, except that material may be duplicated by you for your research use or educational purposes in electronic or print form. You must obtain permission for any other use. Electronic or print copies may not be offered, whether for sale or otherwise to anyone who is not an authorised user. 


\title{
Interpersonal Coordination in Dyadic Performance
}

\author{
Marc R. Thompson', Yorgos Diapoulis', Tommi Himberg' \& Petri Toiviainen'
} 'Music Department, University of Jyväskylä, Finland

2Department of Neuroscience and Biomedical Engineering, Aalto University, Finland

\begin{abstract}
Dyadic musical performance provides an excellent framework to study interpersonal coordination because it involves multiple agents performing matched, rhythmic and/or interactive behaviors. In this chapter, we explore interpersonal coordination using Canonical Correlation Analysis as a coupling measure. To provide some context when interpreting the output of CCA, musicians performed using different expressive manners (deadpan, normal, exaggerated). Overall the results showed the normal performances were slightly more interpersonally coordinated than deadpan and exaggerated.
\end{abstract}

\section{Introduction}

Dyadic musical performance provides an excellent framework to study interpersonal coordination because it involves multiple agents performing matched, rhythmic and/or interactive behaviors (Keller, 2008; Himberg \& Thompson, 2011). Such behaviors fall under the category of entrainment. As Tommi Himberg explains in chapter XX of this book, the criteria of an entrained process are flexibility (adapting one's behavior to match the behavior of their performing partner), autonomy (a performance may continue even if one violinist makes a mistake or stops playing), and coupling (bi-directional communication leading to matched behavior). The advent of motion capture technology has brought with it a growing interest in developing measures that can identify entrained processes computationally. In this chapter we present the results of a small methods-based study in which we explore how Canonical Correlation Analysis (CCA) can be used as a measure of interpersonal coordination within violinist dyads. Because CCA identifies linear relationships between sets of data, it is well suited to measure the extent to which musicians synchronize their behavior when performing. As this is a novel application of CCA, we will examine its results within 
32 the context of the Total Kinetic Energy (TKE) employed by the musicians during a 33 performance. Like CCA, TKE is computed from motion capture data and provides a global 34 indicator of performance behavior.

36 Let's begin with a small thought experiment that we hope will explain the rationale for using 37 CCA. Suppose that you are attending a concert (it could be a classical ensemble, rock show, jazz quartet, anything). Walking out of the concert you are accosted by an intrepid young music researcher and asked to rate on a scale from 1 to 7 the amount of physical interpersonal coordination displayed between the performers. You give a response that from your perspective best sums up the performance. As a follow-up, the researcher wants to know how you arrived to your numerical response and asks you to dissect the coordination in terms of body parts. Were the musicians' heads moving in synchrony? Were the bowing gestures coupled? Perhaps the musicians had similar swaying patterns? Did the musicians communicate tempo changes with specific gestures? Were the musicians tapping their feet to keep the beat? Because of the sheer number of events occurring simultaneously, you might feel hard-pressed to describe exactly which parts of the body were chiefly responsible for the perceived coordination. One explanation for this is that while you were not paying attention to individual parts of the body, your mind formed a gestalt of the performance and your rating of interpersonal coordination was based on the performance as a whole, not its constitutional elements.

The aim of this thought experiment is to suggest that we are good at recognizing interpersonal coordination between musicians even though we are unable to keep track of every piece of information. With technologies such as optical motion capture, we are able to amass vast amounts of data and compare the movement trajectories of markers attached to two musicians in countless ways. We can for instance, create a correlation matrix that compares all markers in all combinations, and observe that Violinist 1's left elbow velocity is highly synchronized with Violinist 2's right knee velocity. However, just as it is more cognitively economical for humans to construct a gestalt that captures the performance as a whole, it is more efficient to compare higher-level movement features that summarize the violinists' movements as a whole. The great advantage of a coupling measure such as CCA is 
that we are not comparing marker $\mathrm{x}$ with marker $\mathrm{y}$, but comparing Violinist 1 to Violinist 2.

64 Hence, we can compute the relationship between musicians as a whole with a single calculation.

66

To explain how CCA works, it might be useful to begin with a related technique: Principal Components Analysis (PCA). Consider an $m \times 3 n$ matrix of motion capture data whereby $3 n$ represents the number of markers attached to a violinist in a three-dimensional coordinate system, and $m$ is the number of observations (in the current case, this number would be the motion capture's sampling rate (120) times the duration of the performance in seconds). Collectively, the data set contains fingerings, bowings (i.e. movements involved in sound production) as well as head gestures and torso swaying (i.e. movements that enable soundproducing movements). However, much of the data is co-varying (i.e. the head marker's motion is anchored to that of the shoulder markers). PCA is a method that eliminates redundancy embedded within data by creating a new matrix of synthetic orthogonal variables (called the principal components). These variables are linear combinations of the original variables and ordered according to decreasing proportions of variation within the original data matrix (Toiviainen, Luck \& Thompson, 2010). The majority of the variation is contained within the first few variables and the remaining variables can be discarded as noise. When applied to motion capture, PCA provides a higher-order movement feature, one that describes the violinist's global dynamic motion rather than the individual markers' motion. As already mentioned, the variables are orthogonal, meaning that they are non-overlapping and uncorrelated. For motion capture data, this means that each principal component will represent the movement occurring on a single axis. For example, if a violinist's predominant movement pattern is side-to-side swaying, the first principal component will include loadings from any marker that has high variation on the mediolateral axis. The extent to which how many additional components should be retained depends on the complexity of the violinist's movements.

CCA is similar to PCA but takes into account two data matrices, and computes their shared variance. In our case, the matrices represent the movements of each violinist. As such, CCA produces two synthetic data sets. These synthetic variables (called the canonical components) 
94 reflect the variance that is shared with the other matrix. The canonical components are ordered according to decreasing amounts of shared variance; the first canonical components from each data set contain the highest amount of shared variance to maximize their correlation. Like PCA, only the first few canonical components should be interpreted as meaningful, and the remaining can be discarded as noise. In relation to the current study, CCA offers two primary advantages (Sherry \& Henson, 2005). First, as a multivariate technique, it greatly diminishes the probability of committing Type I errors. Correlating everything with everything is likely to produce false positives-CCA decreases the chances of this happening because it is based on a single correlation. Second, CCA enables one to observe how multiple variables interact with each other. Human behavior is complex and governed by multiple causes and effects. While univariate methods test each variable in isolation (i.e. how marker $\mathrm{x}$ is related to marker $\mathrm{y}$ ), CCA offers the potential to examine multivariate relationships (i.e. how Violinist 1 is related to Violinist 2). CCA has previously been used in studies using motion capture and music to investigate relationships between sound-tracing gestures and computationally extracted acoustical features (Caramiaux,

109 Bevilacqua \& Schnell, 2010; Nymoen et al, 2013). In contrast, we are using CCA to identify

110 linear relationships between the gestures of performing musicians, and have not taken into 111 account any acoustical features.

113 In this chapter, we also take into account the coordination of the violinists' affective states 114 and how this might affect the results of the CCA. Research targeting the relationship between 115 affective states and body movement has come of age in the past 20 years, particularly in 116 studies on solo performance. Starting with Davidson's seminal 1993 study, a popular design 117 has been to instruct musicians to perform with varying expressive manners (Davidson, 1993; 118 Palmer et al., 2009; Wanderley et al., 2005; Huang \& Krumhans1, 2011; Thompson \& Luck, 119 2012)-manners might include: deadpan (i.e., without expression), projected (i.e., with 120 normal levels of expression), and exaggerated (i.e., with exaggerated levels of expression). 121 Davidson's (1993) study focused on the perception of expressive manner revealed that less 122 experienced observers were more likely to use the visual modality when rating level of 123 musical expressivity. More recent perceptual studies have further investigated the crossmodal 124 interactions of vision and sound that observers employ when making expressivity judgments 
125 (Vuoskoski et al., 2013). The 'levels of expression paradigm' has also been used in music 126 production experiments in which the goal has been to analyze the kinematics of pianists 127 whose movements have been motion captured. Thompson and Luck's (2012) data showed

128 that the head and shoulders had bigger differences between expressive manners, compared to

129 the fingers and wrists. The authors argued that pianists might use the parts of the body not 130 involved in sound production as a means of conveying expressivity, as these would have 131 greater degrees of freedom than parts of the body directly involved in sound production. In 132 recent years, an increased accessibility of sensor technologies, as well as an advancement 133 towards studying music performance from the lens of embodied cognition (Leman, 2007), 134 has prompted a number of studies on interpersonal coordination within musical dyads to use 135 motion capture for data collection (Goebl \& Palmer, 2009; Keller \& Appel, 2010; also see 136 Repp \& Su, 2013).

138 The Current Study

139 Our aim for this chapter is to contribute to work on interpersonal coordination in dyadic 140 performance by using canonical correlation analysis (CCA) for quantifying coupled 141 movements in a musical performance. Below we report a small study in which three violinist 142 dyads were motion-captured while performing a short piece. Our focus is on non-sound143 producing gestures (sometimes called ancillary gestures; Wanderley et al., 2005). Ancillary 144 gestures are best represented through low-level kinematic features such as velocity. For a 145 coupling measure like CCA, velocity is more appropriate than position for two main reasons. 146 First, it eliminates the position factor. For instance, if one waves their hand at two different 147 locations, the velocity data are similar while the position data are dissimilar. Second, velocity 148 is mostly stationary (e.g. zero-centered), whereas position may not be. Nonstationarity in 149 position data (e.g. a significant change of posture) may be the single determining factor in 150 correlation, which may hide other, possibly important but more nuanced forms of coupling. 151 Because CCA is novel in this context, we compare its results with Total Kinetic Energy, 152 which has been used previously to summarize performance behavior (Toiviainen et al., 153 2010). As to the experimental design, we felt it would be useful to examine how the results 154 of canonical correlation analysis might vary under different performance conditions. Because 155 our previous work has shown that musicians alter their movement patterns when requested to 
156 perform in different levels of expression (Thompson \& Luck, 2012), and in different tempi

157 (Thompson et al., 2015), we hypothesized that interpersonal coordination would also be

158 different according to instructed expressive intention and tempo, and that these differences

159 would be reflected in the results of canonical correlation analysis.

160

161 Method

162 Participants

163 Three violin dyads participated in this study (6 musicians total; 4 females; age: $M=24.1, S D$

$164=1.7)$. The violinists were recruited from student populations at the University of Jyväskylä

165 and JAMK University of Applied Sciences. Musicians had received on average 15.8 (SD =

166 2.3) years of instrumental training on the violin. Although the violinists knew each other,

167 none had spent any significant amounts of time performing together before to the experiment.

169 Procedure

170 The dyads performed a short piece arranged for two violins: De Kleinste, composed by J.

171 Beltjens (16 bars, 6/8 time signature). Each dyad performed the piece nine times in a $3 \times 3$ task

172 design: three expressive manners (deadpan, normal, exaggerated) performed using three

173 tempi (60-BPM, 90-BPM, free tempo). Our data set thus consists of 27 performances $\left(M_{\text {duraion }}=\right.$

$\left.17433.1 \mathrm{sec}, S D_{\text {duration }}=4.6 \mathrm{sec}\right)$.

175

176 The tempo was given using a two-measure metronome count-in. Despite instructing a tempo,

177 the dyads gravitated to their own tempo, abandoning the instructed tempo within a few bars.

178 To explore the deviation from the instructed tempo, we estimated the actual tempo for each

179 performance. Due to timing dynamics, the tempi also fluctuated within the performances; to

180 calculate a performance's average tempo, we divided the performance's duration in seconds

181 by the number of eighth note values in the score. We then converted this time in seconds per 182 meter to BPM. Generally, the dyads performed faster than the instructed tempo (actual 60183 BPM: $M=76.56$ BPM, $S D=5.66$; actual 90-BPM: $M=94.78$ BPM, $S D=12.35)$. For the 184 Free Tempo condition, the average tempo was 77.1 BPM $(S D=7.16)$. Because the Free 185 Tempo was so close to the actual tempo for 60-BPM, we estimate that the overall preferred 186 performing tempo was about 76 or 77 BPM (the tempo marking on the score for De Kleinste. 
$18750 \mathrm{BPM}$ for each dotted quarter note). Initial analysis exploration indicated that tempo did not interact in any meaningful way with the expressive manners. Therefore, we eliminated tempo as a factor and all of the results presented below represent data that is averaged by the expressive manner.

\section{Audio Recordings}

193 Audio of the experimental trials was recorded using two AKG C417 L wireless microphones.

194 The microphones were positioned around each violinist's right ear lobe and secured with 195 adhesive tape. Note onsets were annotated from the recorded audio files in Reaper digital 196 audio workstation manually using the marker utilities extension. Each take was exported as a 197 plain text file with note onsets in samples.

\section{Motion Capture}

200 Optical motion capture data was generated using eight Qualisys Oqus cameras at $120 \mathrm{~Hz}$.

201 Twenty-six markers were placed on the joints of each musician, and five markers were placed on the violin ( 2 on the bow, and 3 on the violin itself). The data were labeled within

203 Qualisys' Track Manager software and analyzed in MATLAB using functions within the 204 MoCap Toolbox (Burger \& Toiviainen, 2013). CCA was carried out using MATLAB's canoncorr function.

207 Feature Computation

208 Total Kinetic Energy

209 For every performance we computed the instantaneous kinetic energy for each performer 210 individually based on the method used by Toiviainen et al. (2010). The total kinetic energy 211 was estimated as the sum of the kinetic energy of both performers, which consists of the total 212 amount of translational and rotational energy computed for each marker. Specifically, we 213 used the function mckinenergy from MoCap Toolbox (Burger \& Toiviainen, 2013) to 214 compute both the translational and rotational energy based on a body segment model 215 proposed by Dempster (1959). We then calculated the mean kinetic energy for each marker, 216 and took the grand sum for both performers. Total kinetic energy (TKE) describes the amount 217 of physical activity across both performers in one performance. 
219 Canonical Correlation Analysis: CanCon1 and CanCon2

220 CCA was employed on each performance separately. Each performance produced two 221 matrices (V1 and V2) representing the three-dimensional coordinates of 28 reflective markers 222 attached to each violinist. Because we were interested in coupled ancillary movement (e.g. 223 swaying), we first reduced the data to seven markers representing non-sound producing 224 movement patterns of each violinist: head, left shoulder, right shoulder, neck, left hip, right 225 hip and hip centroid. From the raw three-dimensional location data, we estimated the velocity 226 of V1 and V2 using numerical differentiation. To this end, we applied finite difference 227 followed by a second-order smoothing Butterworth filter with a $0.2 \mathrm{~Hz}$ cutoff frequency (see 228 Burger \& Toiviainen, 2013). As mentioned above, the markers attached to a musician 229 generate data that are largely co-varying. Entering the velocity data from all seven markers 230 into the CCA equates to including noise to the CCA, and results in an over-fitted model. For 231 this reason, Principal Components Analysis (PCA) was applied to V1 and V2 to reduce the 232 number of input variables. On average, we found the first two principal components retained $23385.5 \%$ of the total variance within the raw movement data $\left(M_{\mathrm{PCI}}=72.6 \%, S D_{\mathrm{PC} 1}=14.27 \% ; M_{\mathrm{PC} 2}\right.$ $234=13.26 \%, S D_{\mathrm{PC} 2}=7 \%$,). The transformed data, called the PC scores (original data expressed 235 as values of the reduced set of variables), were entered in the CCA. We name these input 236 variables PCs1 and PCs2.

238 The outputs of CCA are the canonical loadings (A and B), which are the sets of coefficients 239 that indicate the contributions of the variables from PCs1 and PCs2 to the new sets of 240 variables, and the canonical scores ( $\mathrm{U}$ and V), which are the new variables themselves. Like 241 in PCA, the canonical scores are synthetic data sets in which the column variables are 242 orthogonal to each other. Instead of being ordered according to variance within a single set of 243 variables, the canonical scores are ordered according to the variance shared between PCs1 244 and PCs2. Therefore the highest correlation exists between the respective first canonical 245 components (CanCon1) as they contain as much of the shared variance as possible between 246 PCs1 and PCs2. The second canonical components (CanCon2) in turn account for as much of 247 the remaining shared variance as possible. The strength of the relationship between the 248 canonical components is represented by the sample correlation between respective columns 
249 of $\mathrm{U}$ and $\mathrm{V}$. In CCA, the correlation values range from 0 to 1 . A value closer to 1 implies that

250 violinists coupled their movements, or were to some extent synchronized.

251

252 It is critical to mention that when using CCA for timeseries movement data, we must take 253 into account that the data are autocorrelated, and do not meet the assumption of serial 254 independence between observations required for parametric tests. Specifically, the sample 255 correlation between two variables containing autocorrelated observations has fewer degrees 256 of freedom than two variables containing serially independent observations. In other words, 257 in a timeseries, the number of observations that are free to vary is presumably less than $N-1$, 258 where $N$ is the number of observations. To overcome this obstacle, we estimated the effective 259 degrees of freedom based on the autocorrelation functions of $U$ and $\mathrm{V}$, using a formula that 260 takes into account the autocorrelation coefficient appearing at the maximum lag (Pyper and 261 Peterman, 1998; Alluri et al., 2012). Averaged for all 27 performances, we calculated the 262 effective degrees of freedom for CanCom1 $\left(M_{\mathrm{ccl}}=47.28, S D_{\mathrm{ccl}}=27.42\right)$ and CanCom2 $\left(M_{\mathrm{cc} 2}=\right.$ $\left.263116.96, S D_{\text {сс2 }}=187.23\right)$, which were in turn used to compute the correlation $p$-values. For 264 CanCon1, all performances were significant $(p<.05)$, whereas for CanCom2, none of the 265 performances were significant. 

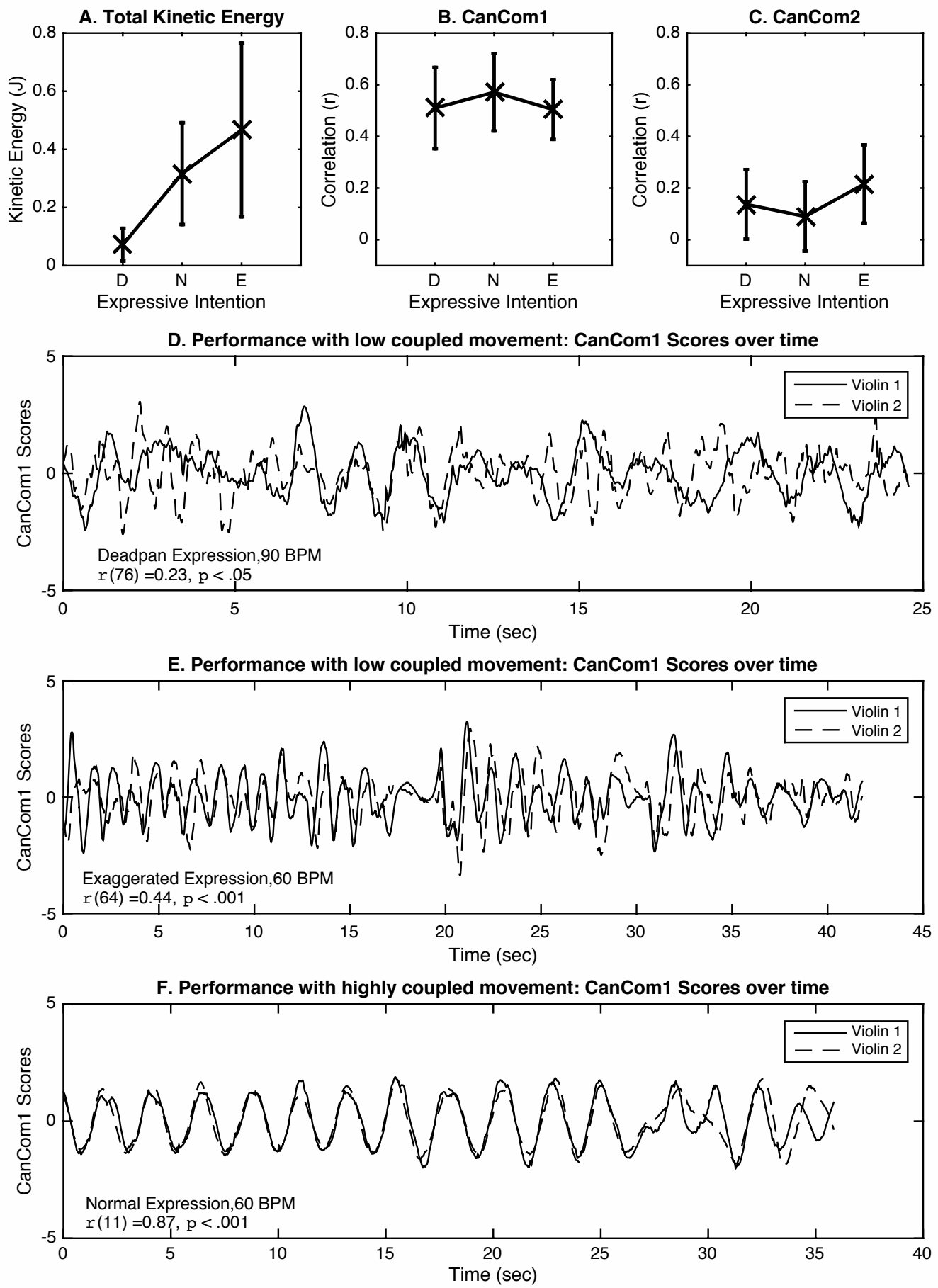
284 Feature Comparison

285 Total Kinetic Energy: Figure X, panel A shows the mean TKE for deadpan, normal and 286 exaggerated performances, with error bars to represent standard deviation. The results show 287 that different expressive manners are reflected in the total amount of movement, with greater 288 amount of movement observed as expression level increases (deadpan: lowest, exaggerated: 289 highest). These results can be viewed as a proof of concept for the expressive manners: 290 Musicians altered their behavior when asked to perform with more expression. It should be noted that the musicians were never overtly instructed to change their movement patterns according to the expressive manner. Rather, the different behaviors are natural emergent consequences of the expressive manners.

CanCom1 \& CanCom2: Panels B and C show the mean sample correlations for deadpan, normal and exaggerated for CanCom1 and CanCom2 respectively. Panel B shows that each expressive manner was performed with roughly similar amounts of coupled movements. However, normal performances are slightly more coupled than the other two. One interpretation is that normal performances did not require extra cognitive load on the part of the players. That is, they could perform without taking into consideration what it meant to play deadpan or exaggerated.

303 Panels B and C also indicate information regarding movement complexity. It appears that the 304 shared variance within deadpan and normal performances is essentially entirely explained by 305 CanCom1. This could be interpreted by stating on average, interpersonal coupling in these performances was occurring on a single axis. In other words, the movement associated with coupling was not complex. For the exaggerated performances, the gap is smaller between

308 CanCom1 and CanCom2, meaning that the shared variation is distributed across the two canonical components. Taking into account that exaggerated performances possessed the most kinetic energy (panel A), we can make the assertion that the interpersonal coordination

311 in the exaggerated performances was generally more complex, and occurring on multiple 312 axes. 


\section{Exploring the Canonical Loadings and Canonical Scores}

315 Now let us examine more closely the canonical loadings and canonical scores. For this 316 section, we have singled out performances that best exemplify each expressive manner. We

317 recommend the reader to watch the performances in this chapter's accompanying video

318 (found at https://www.youtube.com/watch?v=fvv2-he5lco\&t=67s ).

320 Given that we have performed canonical correlation analysis on the first two principal components of seven velocity features that were estimated from three-dimensional marker location data (gasp!), you may be asking the very sensible question: After so much processing, how do the canonical scores and loadings relate to the original motion capture data? To answer this question, let us first consider what information the loadings and scores provide. Since we are dealing with motion capture data, an intuitive way to think about the loadings and the scores is that the scores provide temporal information while the loadings provide spatial information.

Canonical scores: The canonical scores represent temporal information because they are

330 timeseries with the same amount of observations as the original location data. If we overlay

331 the canonical scores from each violinist, we get an impression as to how the coupling evolves

332 throughout the performance. In panels D, E and F, we have plotted the CanCom1 canonical 333 scores from performances that best represent low and high gesture coupling. Panel D shows 334 the canonical score from the deadpan performance with the lowest CanCom1 value $(r=.23)$ 335 out of the 27 performances (first lowest overall). Panel E shows the canonical score from the 336 exaggerated performance with the lowest CanCom1 value $(r=.44)$ out of the 27 337 performances (sixth lowest overall). Panel F shows the normal performance with the highest

338 CanCom1 value $(r=.87)$ and highest over all 27 performances. Comparing the three panels, 339 we see that the deadpan performance scores are irregular and do not co-vary coupling in the 340 normal performance (panel F) is regular with some deviations after the 25-second mark. 341 Meanwhile, the

343 Canonical loadings: Recall that the loadings indicate the various contributions of the input 344 variables (PCs1 and PCs2) to the output variables (U and V). These contributions can be 
inspected visually through the use of animations. In our accompanying video, we have created animations that represent the loadings of CanCom1 and CanCom2 for each example performance. The animations were created by projecting the canonical loadings back to the

348 kinematic (velocity) space by multiplying the PC loading matrix with the loading matrix of 349 the two canonical components and adding the mean position back to the data. The animations

350 should not be seen as being time-dependent; they demonstrate the extent to which the various markers contribute to the canonical components. The extent to which a marker is moving indicates its contribution to the canonical component. The animations also demonstrate how canonical components are orthogonal, and so the variability within a canonical component is largely restricted to a single axis.

\section{Conclusions}

358 At the beginning of this chapter, we mentioned that an entrained process should contain flexibility, autonomy and coupling. In truth, employing CCA the way we did does not allow us to identify entrained processes because we cannot account for flexibility and autonomy. While CCA provides a measure of linear coupling, it only gives a global measure, ignoring the time-dependent aspects of entrained processes. Our earlier studies on entrainment in dance performance within dyads (Himberg \& Thompson, 2010; Himberg \& Thompson, 2011) found that expert/novice dyads remained synchronized not by appropriating a leader/follower dynamic, but through mutual adaptation in response to each other's movements. This does not rule out that entrainment never occurred within our data set. On the contrary, looking at panels D and E, we can identify some epochs within the timeseries that appear more together than others. This variation within the canonical scores indicates that performers adapted their behaviors throughout the performance. Therefore, CCA might have the potential to identify entrained processes if for example, the scores were windowed and multiple comparisons were performed laterally on a single time series. Additionally, cross-correlation analysis could identify the direction of the interaction and reveal

373 leader/follower relationships. Using these alterations to our method, we may be able to use

374 CCA more effectively for identifying dynamic entrainment processes. 
376 There are significant drawbacks to CCA for motion capture data. As explained above, the 377 serial correlation within the data requires the use of effective degrees of freedom for 378 calculating $p$-values. The greater amount of serial correlation leads to a lower amount of 379 degrees of freedom (the performance in panel $F$ contains only 11 effective degrees of 380 freedom for over 2000 observations). Another drawback is that CCA can only identify linear 381 relationships between two data sets; it is ineffective for detecting processes that are non382 linear. For detecting non-linear relationships, there exists a broad range of coupling 383 techniques other than CCA. For example, mutual information is a technique that identifies the 384 mutual dependence between two data sets. While mutual information would do a better job of 385 finding non-linear relationships between sets, it is a bin-based approach that requires 386 knowing how many bins to use before the calculation. As such, the result is greatly affected 387 by input parameters. In this respect CCA is advantageous because it is parameter-free and 388 simpler to compute.

390 To summarize, we have examined Canonical Correlation Analysis as a means of quantifying 391 coupled movement in performing dyads. To provide a context when interpreting the output of 392 CCA, musicians performed using different expressive manners (deadpan, normal, 393 exaggerated). We also calculated the total kinetic energy across the expressive manners, 394 which provided evidence that musicians altered their behavior based on the performance 395 instruction. Overall the results showed the normal performances were slightly more 396 interpersonally coordinated than deadpan and exaggerated. While we cannot claim that our 397 exact results would be replicated (music performance is an idiosyncratic endeavor), we hope 398 we have presented CCA in sufficient detail to inspire others interested in interpersonal 399 coordination to explore this coupling measure. 
402

403

404

405

406

407

408

409

410

411

412

413

414

415

416

417

418

419

420

421

422

423

424

425

426

427

428

429

430

431

432

\section{References}

Alluri, V., Toiviainen, P., Jääskeläinen, I. P., Glerean, E., Sams, M., \& Brattico, E. (2012). Large-scale brain networks emerge from dynamic processing of musical timbre, key and rhythm. Neuroimage, 59, 677-3689.

Burger, B. \& Toiviainen, P. (2013). Mocap toolbox - a matlab toolbox for computational analysis of movement data. In Bresin, R., (Ed.), Proceedings of the 10th Sound and Music Computing Conference (pp. 172-178), Stockholm, Sweden. KTH Royal Institute of Technology.

Caramiaux, B., Bevilacqua, F., \& Schnell, N. (2010). Towards a gesture-sound cross-modal analysis. In Gesture in Embodied Communication and Human-Computer Interaction, (pp. 158-170). Springer.

Davidson, J. (1993). Visual perception of performance manner in the movements of solo musicians. Psychology of Music, 21, 103-113.

Dempster, W. T., Gabel, W. C., \& Felts, W. J. L. (1959). The anthropometry of manual work space for the seated subject. American Journal of Physical Anthropology, 17, 289-317.

Goebl, W. \& Palmer, C. (2009). Synchronization of timing and motion among performing musicians. Music Perception, 26, 427-425.

Himberg, T. \& Thompson, M. (2010). Dyadic entrainment and interaction in african dance. In Demorest, S., Morrison, S., and Campbell, P., (Eds.) Proceedings of the 11th international conference for music perception and cognition, (ICMPC '11), (pp. 425428), Seattle, USA. University of Washington.

Himberg, T. \& Thompson, M. R. (2011). Learning and synchronizing dance movements in South African songs: Cross-cultural motion-capture study. Dance Research, 29(special electronic issue), 305-328.

Hotelling, H. (1936). Relations between two sets of variates. Biometrika, 28, 321-377.

Huang, J. and Krumhansl, C. L. (2011). What does seeing the performer add? It depends on musical style, amount of stage behavior, and audience expertise. Musica Scientiae, 15, 343-364.

Keller, P. E. (2008). Joint action in music performance. In Morganti, F., Carassa, A., and Riva, G., (Eds.) Enacting Intersubjectivity: A Cognitive and Social Perspective on the Study of Interactions, (pp. 205-221). IOS Press, Amsterdam. 
Keller, P. E. (2013). Ensemble performance: Interpersonal alignment of musical expression. In Fabian, D., Timmers, R., and Schubert, E., (Eds.), Expressiveness in music performance: Empirical approaches across styles and cultures. Oxford University Press.

Keller, P. E. \& Appel, M. (2010). Individual differences, auditory imagery, and the coordination of body movements and sounds in musical ensembles. Music Perception, 28, $27-46$.

Leman, M. (2008). Embodied music cognition and mediation technology. Cambridge, MA: MIT Press.

Nymoen, K., Godøy, R.I., Jensenius, A.R., \& Torresen, J. (2013). Analyzing correspondence between sound objects and body motion. ACM Transactions on Applied Perception, 10, article $9,1-22$.

Palmer, C., Koopmans, E., Carter, C., Loehr, J., \& Wanderley, M. (2009). Synchronization of motion and timing in clarinet performance. In Williamon, A., Pretty, S., and Buck, R., (Eds.), Proceedings of the International Symposium on Performance Science, (pp. 159164). European Association of Conservatories (AEC), Utrecht.

Pyper, B. J. \& Peterman, R. M. (1998). Comparison of methods to account for autocorrelation in correlation analyses of fish data. Canadian Journal of Fisheries and Aquatic Sciences, 55, 2127-2140.

Repp, B. H. \& Su, Y.-H. (2012). Sensorimotor synchronization: A review of recent research (2006-2012). Psychonomic Bulletin \& Review, 20, 403-452.

Sherry, A. \& Henson, R. K. (2005). Conducting and interpreting canonical correlation analysis in personality research: A user-friendly primer. Journal of Personality Assessment, 84, 37-48.

Thompson, M. R., Diapoulis, G., Johnson, S., Kwan, P. Y., \& Himberg, T. (2015). Effect of tempo and vision on interpersonal coordination of timing in dyadic performance. In Aramaki, M., Kronland-Martinet, R., and Ystad, S., (Eds.), Proceedings of the 11th International Symposium on Computer Music Multidisciplinary Research. University of Plymouth, UK.

Thompson, M. R. \& Luck, G. (2012). Exploring relationships between pianists' body movements, their expressive intentions, and structural elements of the music. Musicae Scientiae, 16, 19-40. 
464 Toiviainen, P., Luck, G., \& Thompson, M. R. (2010). Embodied meter: Hierarchical 465 eigenmodes in music-induced movement. Music Perception, 28, 59-70.

466 Vuoskoski, J., Thompson, M., Spence, C., \& Clarke, E. (2013). Crossmodal interactions in 467 the perception of expressivity in musical performance. Attention, Perception, \& 468 Psychophysics, 76, 591-604.

469 Wanderley, M. M., Vines, B. W., Middleton, N., McKay, C., \& Hatch, W. (2005). The 470 musical significance of clarinetists' ancillary gestures: An exploration of the field. Journal 471 of New Music Research, 34, 97-113. 